\title{
Transmission Electron Microscopy Study on Platinum Nanoparticle Distribution in PEMFC Catalyst Layers under Different Ionomer/Carbon Ratios Fabricated by Direct Dry Deposition
}

\author{
Haoran $\mathrm{Yu}^{1,2}$, Justin Roller ${ }^{1,3}$, Rishabh Jain ${ }^{1,3}$, C Barry Carter ${ }^{2,3}$, and Radenka Maric ${ }^{1,2,3}$ \\ 1. Center for Clean Energy Engineering, 44 Weaver Road Unit 5233, Storrs, CT 06269-5233 \\ 2. Department of Chemical \& Biomolecular Engineering, University of Connecticut, 191 Auditorium \\ Road, Unit 3222, Storrs, CT 06269-3222 \\ ${ }^{3 .}$ Department of Materials Science and Engineering, University of Connecticut, 7 North Eagleville \\ Road, Unit 3136, Storrs, CT 06269-3136
}

Proton exchange membrane fuel cells (PEMFC) are poised for market penetration in the automobile, portable and back-up power markets. Cost and durability issues are crucial to the performance of PEMFC, which primarily depends on the design of catalyst layers [1]. Current catalysts layers use platinum (Pt) supported on high-surface-area carbons (e.g., Ketjen Black ${ }^{\circledR}$ and Vulcan $\left.{ }^{\circledR}\right)$ with perfluorosulfonic acid (PFSA) ionomer as a binding agent. It is widely believed that incorporation of ionomer can extend the reaction zone, increasing the proton conductivity and catalyst utilization [2]. The catalyst layers are predominately comprised of primary carbon particles, 20-50 $\mathrm{nm}$ in diameter. Stacking of primary carbon aggregates creates a unique 3D structure between the fractal structured carbon branches [3]. Depositing the carbon with Pt nanoparticles can block these micropores since the size of the Pt particles ranges between 2-4 nm. The PFSA ionomer is distributed on the surface of carbon agglomerates and covers intra-agglomerate pores $(<20 \mathrm{~nm})$ at lower ionomer content, e.g., below $30 \mathrm{wt} \%$ ionomer. Significant blocking of the pores is observed with higher ionomer content ( $>50 \% \mathrm{wt}$.) [4].

Although researches have been focused on influence of ionomer/carbon ratio in catalyst layer microstructure, few have truly revealed the mechanism of how Pt nanoparticles are incorporated into the carbon matrix and the reason for certain ionomer/carbon ratio exhibit better electrochemical performance and higher catalyst utilization [4]. Transmission electron microscopy (TEM) is an advantageous tool for investigating the spatial distributions of components even for complicated functional materials, and can be used to analyze the composition of a local area at a nano- or atomicscale. For example, using TEM combined with electron energy loss spectroscopy (EELs) and energydispersive X-ray spectroscopy (EDS), researchers characterized Pt binary alloys for fuel cell catalysts and investigated the interaction with catalyst support materials. Oezaslan et al. discovered the existence of different morphology regimes in dealloyed $\mathrm{Pt}-\mathrm{Co}$ and $\mathrm{Pt}-\mathrm{Cu}$ particle ensembles, a core-shell structure of nanoparticles with $10-30 \mathrm{~nm}$ in diameter [5]. The interaction of $\mathrm{Pd}_{3} \mathrm{Pt}_{2}$ alloy nanoparticles with the carbon support was studied by Slanac et al. [6]. Aberration corrected STEM revealed a corrugated crystalline alloy core surrounded by a disordered alloy shell that created strong electronic binding of Pd to the 20-50 $\mathrm{nm}$ carbon particles, facilitate strong wetting on the carbon at the particle-support interface, which significantly enhanced the mass activity for oxygen reduction reaction (ORR) [6]. In addition, TEM also helps investigating the change of catalyst microstructure during electrochemical test. Akita et al. have studied Pt nanoparticle distribution before and after membrane electrode assembly (MEA) test and an aggregation process during MEA testing was found under high resolution TEM [7].

Herein we apply a novel depositing method, the Reactive Spray Deposition Technique (RSDT) for catalyst layer fabrication from vapor phase [8]. RSDT, a one-step, open-atmosphere dry deposition, is 
able to make thin porous catalyst films with reduced platinum loading. In this process, platinum 2,4pentanedionate is dissolved in xylene and liquefied propane and combusted through an atomizing nozzle. The droplets then burn through the flame forming metallic Pt nanoparticles. These Pt nanoparticles can nucleate homogeneously during time-of-flight or heterogeneously onto the carbon support. The carbon and ionomer are sprayed, as slurry, radially into the post combustion zone by secondary nozzles. Simultaneous deposition of $\mathrm{Pt}$, carbon and ionomer occurs in-flight. Catalyst layer growth occurs via ballistic impact and sticks on a cast Nafion ${ }^{\circledR}$ membrane [8].

Two types of carbon blacks with high surface area, Ketjen Black ${ }^{\circledR}$ EC-600JD and Vulcan ${ }^{\circledR}$ XC-72R, have been applied in our study. We prepare catalyst layers with different ionomer/carbon ratios ranging from $0.05,0.16,0.5,0.8$ to 1 (in weight). Catalyst layers are directly deposited on TEM grids assembled onto specially made substrates for $30 \mathrm{~s}, 60 \mathrm{~s}, 120 \mathrm{~s}$, and $240 \mathrm{~s}$ in order to observe the film growth. TEM images and diffraction patterns of $\mathrm{Pt}$ nanoparticles under different ionomer/carbon ratios are taken and compared. Figure 1 shows the bright field TEM image of $\mathrm{Pt}$ nanoparticles on carbon with ionomer/carbon ratio 0.16. Pt nanoparticles (black dots) are quite uniformly distributed on carbon. The selected-area-diffraction patterns (SADPs) are shown in Figure 2 and rings are indexed with corresponding planes. Moreover, glassy carbon disk electrodes are deposited for $30 \mathrm{~min}$ for electrochemical study. From cyclic voltammetry and polarization curves, the electrochemical chemical surface area (ECSA) and ORR mass activity of different ionomer/carbon ratios have been compared. The results of Pt distribution and electrochemical performance are correlated to explain the relation of mechanism and performance [9].

\section{Reference:}

[1] Y. Wang, et al. Applied Energy (2011) 88, 981-1007.

[2] T. Soboleva, et al. Applied Materials \& Interfaces (2011) 3, 1827-1837.

[3] Z. Yu, et al. Fuel Cells (2012) 12, 557-565.

[4] T. Soboleva, et al. Applied Materials \& Interfaces (2010) 2, 375-384.

[5] M. Oezaslan, et al. J. Am. Chem. Soc. (2012) 134, 514-524.

[6] D. Slanac. Electrochimica Acta (2012) 64, 35- 45.

[7] T. Akita, et al. Journal of Power Sources (2006) 159, 461-467.

[8] J. Roller, et al. Journal of Material Science (2012) 47, 4604-4611.

[9] The authors acknowledge funding from the Department of Energy, Grant number EC-013088-03

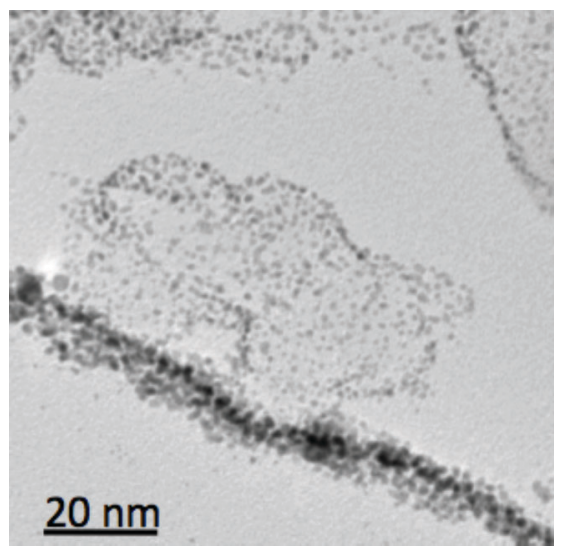

Figure 1. TEM bright field image of Platinum on Carbon.

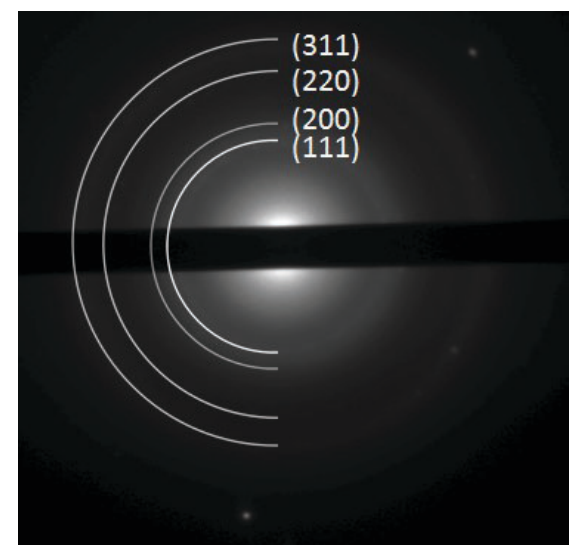

Figure 2. SADPs of Platinum on Carbon. 